\title{
DARK CURRENT AND X RAY MEASUREMENTS OF AN 805 MHZ PILLBOX CAVITY
}

\author{
J. Norem ${ }^{\#}$, ANL, Argonne Il 60439 USA \\ P. Gruber, CERN, Geneva, Switzerland \\ A. Bross, S. Geer, A. Moretti, Z Qian, FNAL, Batavia IL 60510 USA \\ D. M. Kaplan, Y. Torun, IIT, Chicago IL 60616 USA, \\ R. Rimmer, Jefferson Laboratory, Newport News VA 23606 USA \\ Derun Li, M. Zisman, LBNL, Berkeley CA 94720 USA
}

\begin{abstract}
The muon cooling systems proposed for neutrino factories require low frequency (201 MHz) RF cavities with Be windows, at high gradient $\left(\mathrm{E}_{\mathrm{acc}} \sim 16 \mathrm{MV} / \mathrm{m}\right)$, in strong solenoidal magnetic field $(\sim 5 \mathrm{~T})$. For the proposed Muon Ionization Cooling Experiment (MICE) [1], an experimental demonstration of cooling, we have an additional constraint that we must operate sensitive particle detectors very close to the RF cavities, which produce backgrounds from dark currents and $\mathrm{x}$ rays. To understand the processes involved in cavity conditioning and operation near particle detectors, we have constructed a test facility at Lab $\mathrm{G}$ of Fermilab, where a 5 Tesla superconducting solenoid, a $14 \mathrm{MW}$ peak power klystron and a pillbox test cavity at $805 \mathrm{MHz}$ are available. We present measurements of dark currents, $x$ rays and surface structure from the pillbox cavity, with both copper and beryllium endplates, and discuss the interaction between surface structure and radiation backgrounds produced.
\end{abstract}

\section{INTRODUCTION}

Muon cooling systems are necessary for both muon colliders and muon based neutrino beams. While extensive modeling has been done on the design of these systems, the experimental program to study how well the components will operate is just beginning. This paper reports results from tests of an $805 \mathrm{MHz}$ prototype if cavity for a cooling channel.

Muon cooling systems consist of alternating liquid hydrogen absorbers and rf cavities. Since multiple scattering tends to heat rather than cool the beam, and muon beams do not interact strongly with matter, we have assumed that the rf cavities could use windows of beryllium, which produces a higher accelerating field for a given surface field. In order to test how cavities of this type would operate, we have constructed an $805 \mathrm{MHz}$ pillbox cavity with removable end windows in which Be and other materials can be tested. The cavity is basically a cylinder, powered through an arc shaped slot near the outer radius. There is an inner pair of windows that defines the rf properties of the system and an outer pair of windows that maintains the vacuum. A variety of windows have been used on the cavity.

\footnotetext{
\# norem@anl.gov
}

We are looking at a number of issues. We want the maximum gradient we can obtain in these cavities, particularly with Be windows, and the minimum flux of dark current electrons and x-rays that can be produced. We are also interested in how Be windows perform and in information on how $201 \mathrm{MHz}$ cavities will operate with real beams.

\section{DESCRIPTION}

The cavity is shown in Figure 1. The parameters of the different windows are given in the following table and in ref [2].

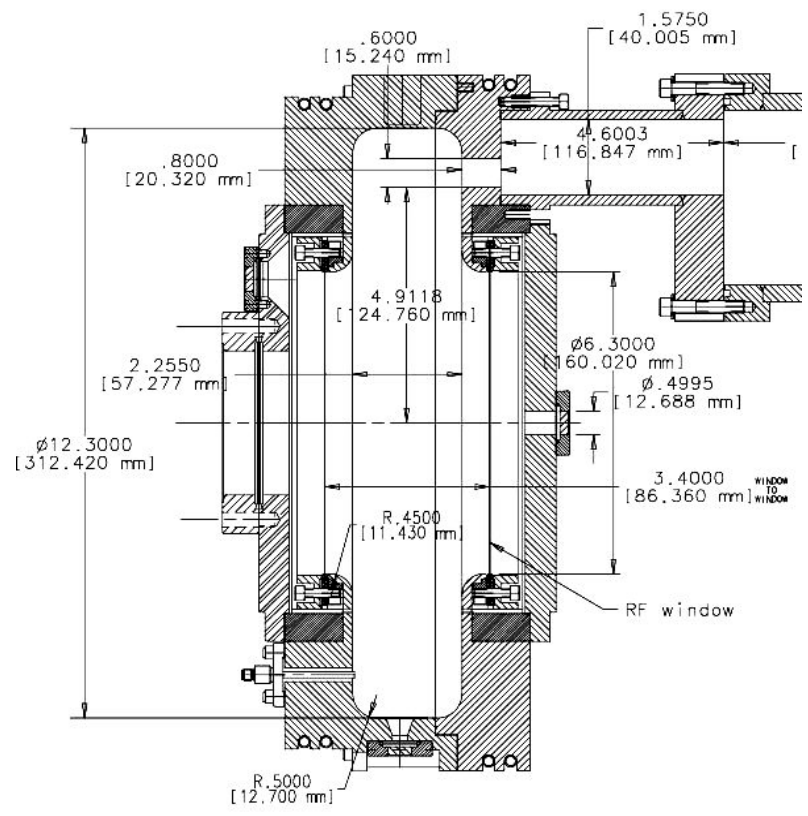

Figure 1. The pillbox cavity.

Window configurations are shown in the table below:

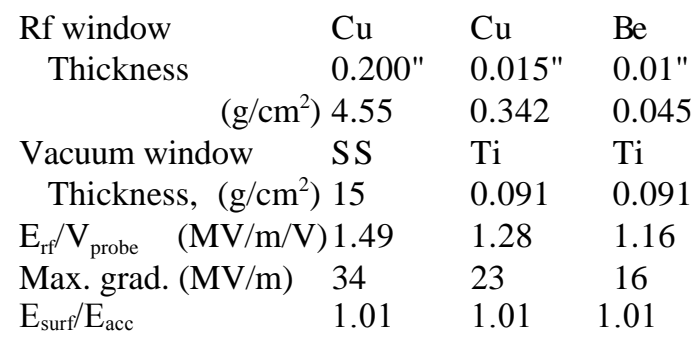




\section{Timeline}

The timeline for experiments with the closed cell cavity is given below. The cavity was first operated with thick of and vacuum windows. The rf components were replaced with thin copper, then Be windows.

1/4/02 Open Cell cavity removed from the Magnet

3/13/02 Begin conditioning with thick $\mathrm{Cu}$ plates

Eventual operation at $34 \mathrm{MV} / \mathrm{m}$, little sparking

7/16/02 Removal of thick Cu plates, little damage

7/30/02 Conditioning with thin $\mathrm{Cu}$ plates to $24 \mathrm{MV} / \mathrm{m}$

9/27/02 Operation in B Field, high BG, $E_{\max }=16 \mathrm{MV} / \mathrm{m}$ Improvements from conditioning with $\mathrm{B}=0$

12/5/02 Removal of thin plates, considerable pitting

12/19/02 Conditioning of TiN/Be windows to $21 \mathrm{MV} / \mathrm{m}$

2/10/03 Begin conditioning with B field Conditioning to $17 \mathrm{MV} / \mathrm{m}$, stable at $14 \mathrm{MV} / \mathrm{m}$

4/22/03 Replacement of Be windows

\section{EXPERIMENTAL RESULTS}

The experimental program has been defined by the particular plates which were installed in this cavity and the electric and magnetic fields used. Conditioning periods with a new surface averaged about a month.

\section{Copper and Be Windows}

With copper windows we were able to reach $34 \mathrm{MV} / \mathrm{m}$ acceleration gradient without a solenoidal field, with very low $\mathrm{x}$-ray, dark current and sparking rates, indicating that the cavity could easily have operated at higher field. Both thick and thin copper plates operated similarly.

When a magnetic field was applied, dark currents, x-ray and sparking rates increased. Fig. 2 shows the measured dark current before the magnet was turned on (bottom band), after the magnet was turned on (top band) and after the magnet was turned off (middle band). These results seem to show that some permanent damage was produced on the windows of the cavity that did not immediately condition away. On the other hand, more extensive conditioning over a one month period with no magnetic field was able to reduce the fluxes of dark currents and electrons to levels seen before the magnetic field was applied.

The Be windows were fabricated by Brush-Wellman. A thin Be foil was supported by a ring of $\mathrm{Be}$. The ring applied a prestress to allow for some heating of the foil without deformation. After annealing, the unit was coated with TiN coating, nominally 150 Angstroms thick, but perhaps thicker. The Be windows conditioned to 16 $\mathrm{MV} / \mathrm{m}$ but over a period of weeks the dark current and sparking rates slowly increased so that it was difficult to return to the initial high accelerating fields.

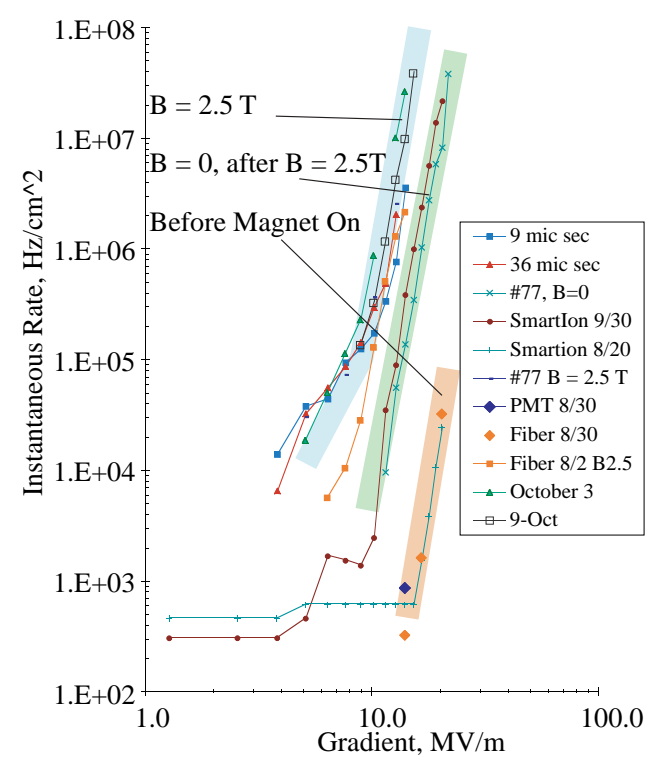

Figure 2. Background rates with copper windows.

Pictures of the dark current transmitted through the windows were recorded on glass slides, polaroid film and photographic paper. The glass and polaroid pictures show the beams near the window in the homogeneous field, and the photographic paper shows how the beams diverge with the fringe fields of the magnets. See a paper by Gruber et al[3].

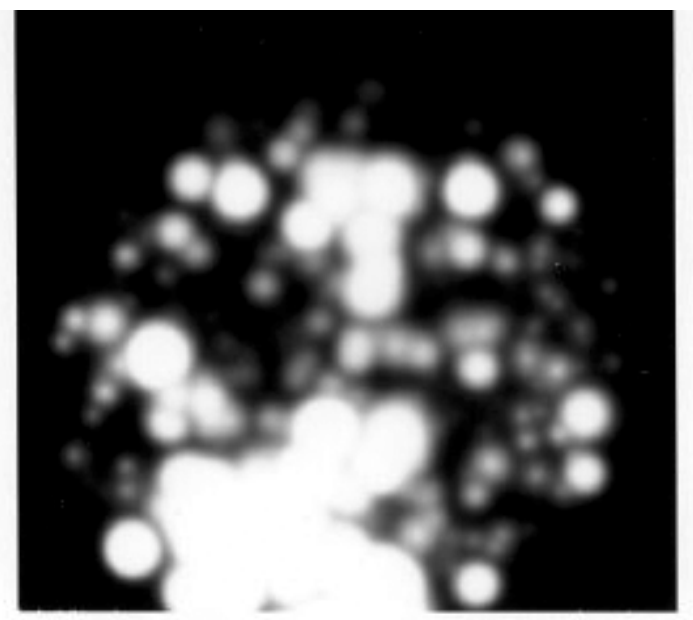

Figure 3. Dark current with 2.5T solenoidal B field.

\section{$X$ ray Spectra}

The spectra of photons were measured with an ORTEC Ge detector, calibrated with Co60 and Cs137. The spectra were obtained at fairly low accelerating fields because the busy gate on the qVt pulse height module was comparable to the length of the rf pulse, so it was not possible to count photons at a rate of more than $1 / \mathrm{rf}$ pulse. The spectrum is somewhat more steep than would be produced from first generation bremsstrahlung, which might indicate that the $\mathrm{x}$-ray energy is being attenuated by scattering. 


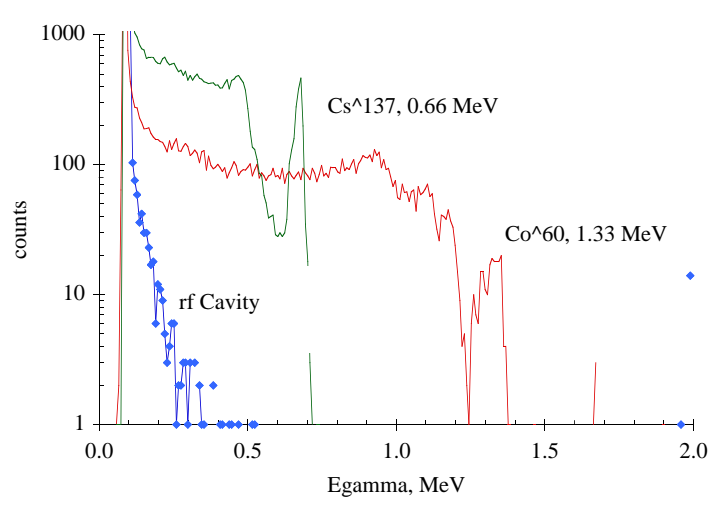

Figure 4. Energy spectra of $x$ rays.

\section{Surface Damage in $\mathrm{Cu}$ and $\mathrm{Be}$}

Surface damage on the $\mathrm{Cu}$ plates consisted of pits on the order of $100-400 \mu \mathrm{m}$ in diameter and occasional splashes where liquid copper drops had evidently splashed and adhered to the surface.

Example of this damage are shown in Fig. 5 and Fig. 6. An electron microscope picture shows a typical sharp edged crater in an otherwise smoothly machined surface. The pits show sharp edges which are consistent with the field emission we see with films and radiation monitors.

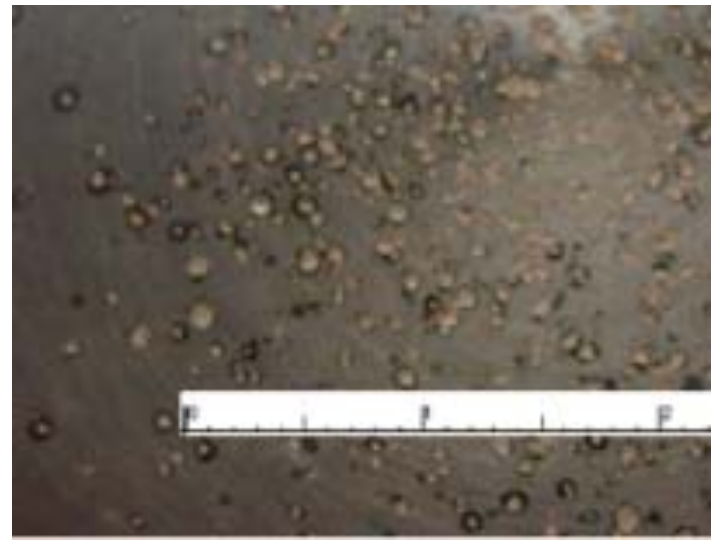

Fig. 5. Optical image of copper plate, (scale in $\mathrm{cm}$ ).

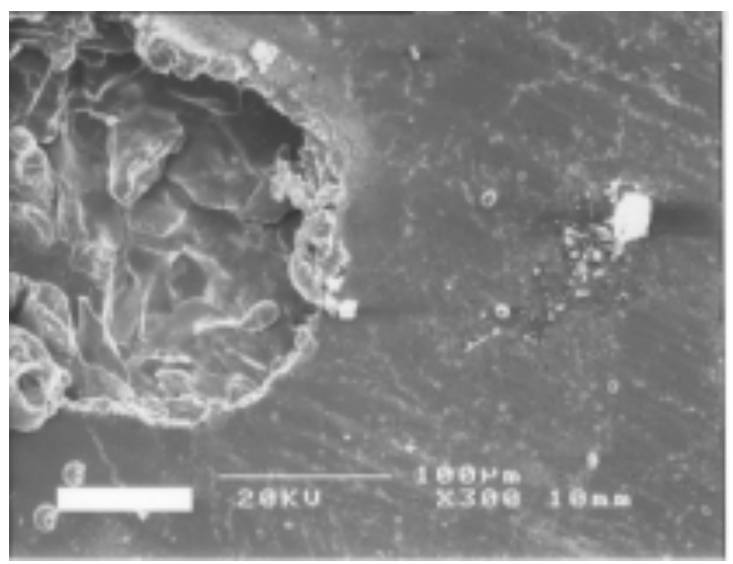

Figure 6. SEM image of copper plate.
The appearance of the Be windows was quite different Visually, the dark TiN coating seemed unbroken, and this seems to be confirmed with electron and optical microscopy, The surface was covered, however, with copper particles. An electron microscope picture of the surface, Fig. 7, shows some of the typical structures. With irregular copper blobs are visible on an otherwise unbroken Be surface. The TiN covered Be shows marks evidently made during rolling. Almost all damage seems to be due to copper deposition, which can be irregular, circular or linear. The $\mathrm{Cu}$ surfaces show admixtures of $\mathrm{Fe}$ at the $2.5 \%$ level when EDX analysis is done, trace amounts of $\mathrm{Fe}$ were in the Be. We also saw deposits of what may have been dried machining or cleaning fluid.

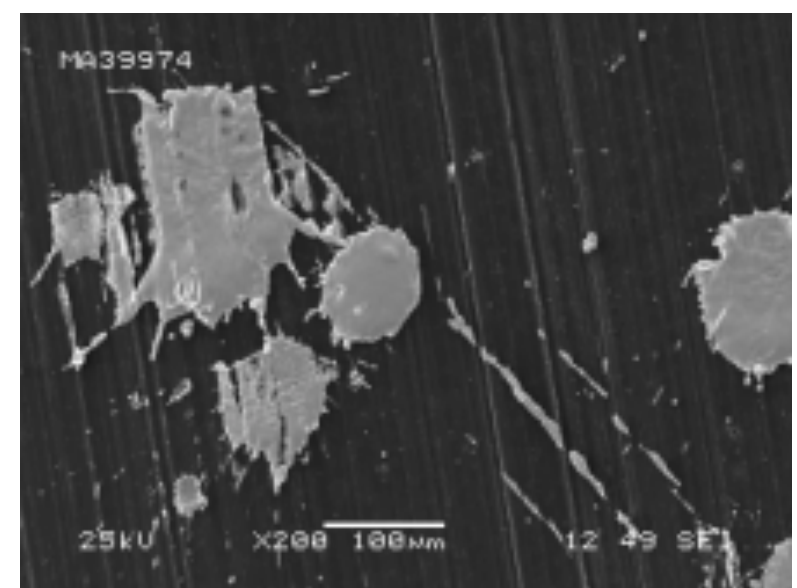

Figure 7. SEM image of Be Windows showing copper.

\section{CONCLUSIONS}

We have built an rf cavity which can be used to study the use of beryllium rf windows which are needed in low frequency cavities for muon cooling We have found that copper cavities seem to produce acceptable background rates at $8 \mathrm{MV} / \mathrm{m}$, however strong $(\sim 2.5 \mathrm{~T})$ magnetic fields can produce problems which require long conditioning periods. The dark current performance of Be windows seemed to degrade with time. A preliminary analysis of the windows themselves, however, using SEM and optical microscopy, shows that all the damage seems to be due copper contamination, and little or no significant damage has been done to the TiN coated Be windows. This work was done as part of the MUCOOL experimental program and was supported by the USDOE and the State of Illinois.

\section{REFERENCES}

[1] http://mice.iit.edu/cooldemo/.

[2] D. Li et al, Tests of an $805 \mathrm{MHz}$ Pillbox Cavity at Lab $G$ of Fermilab, this conference.

[3] P. Gruber, et al. Beam Photography: A Technique for imaging Dark Currents, this conference. 